



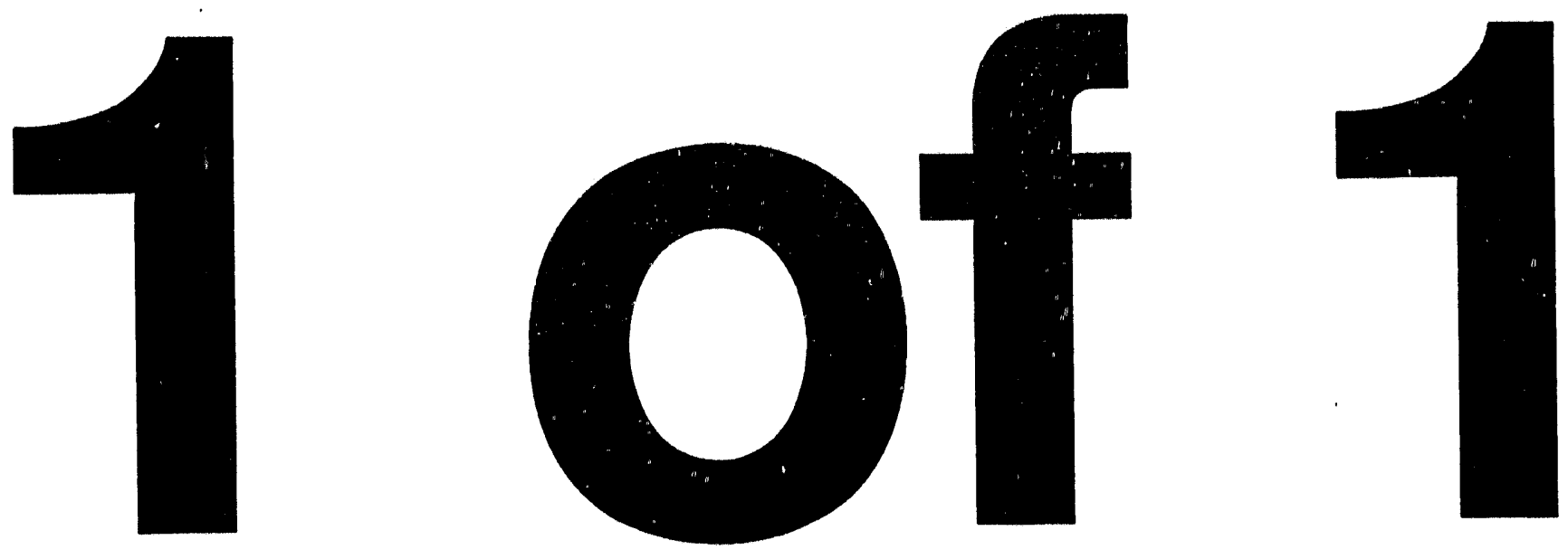


\section{DISCLAIMER}

This report was prepared as an account of work sponsored by an agency of the United States Government. Neither the United States Government nor any agency thereof, nor any of their employees, makes any warranty, express or implied, or assumes any legal liability or responsibility for the accuracy, completeness, or usefulness of any information, apparatus, product, or ence herein to any specific commercial product, process, or service by trade name, trademark. manufacturer, or otherwise does not necessarily constitute or imply its endorsement, recommendation, or favoring by the United States Government or any agency thereof. The views and opinions of authors expressed herein do not necessarily state or reflect those of the United States Government or any agency thereof. process disclosed, or represents that its use would not infringe privately owned rights. Refer-

\section{UEIVED \\ OCT $0>1993$ \\ OSTI}

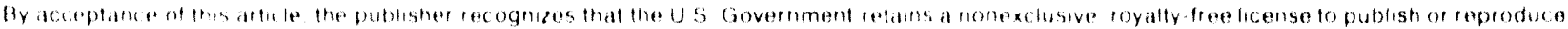

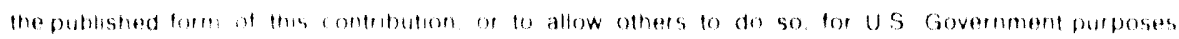

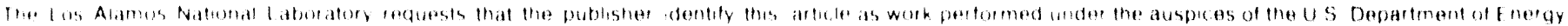




\title{
Calculations of Bose-Einstein Correlations from Relativistic Quantum Molecular Dynamics
}

\author{
J. P. Sullivan ${ }^{\text {a* }}$, M. Berenguer ${ }^{\text {a*}}$, D. E. Fields ${ }^{\text {a* }}$, B. V. Jacak ${ }^{\text {a* }}$, M. Sarabura ${ }^{\text {a*, }}$ \\ J. Simon-Gillo ${ }^{a *}$, H. Sorge ${ }^{\text {a* }}$, II. van Hecke ${ }^{\text {a* }}$, and S. Pratt \\ "Los Alamos National Laboratory, Los Alamos, NM 87545, USA \\ 1. Michigan State University, East Lansing, MI 48824, USA
}

\section{INTRODUCTION}

Bose- Einstein correlation functions which are in good agreement with pion data [1, 2] can be calculated from an event generator [3]. Ilere pion and (preliminary) kaon data from CERN experiment $N \wedge 44[2,4,5]$ are compared to the calculations. 'The dynamic's of 200 GeV/nucleon ${ }^{32} \mathrm{~S}+\mathrm{Pb}$ collisions are calculated, without correlations due to interference patterns of a many-body wavefunction for identical particles, using the Relativistic Quantum Molect!ar Dynamies model (RQMI)) [6]. 'The model is used to generate the phase-space coordinates of the emitted hadrons at the time they suffer their last strong interaction (freeze-out). Ising the freczeout position and momentum of pairs of randomly selected identical particles, a two-particle symmetrized wave-function is calculated and used to add two-body correlations. Details of the technique have been described previously [3]. The method is similar to that used in the Spacer program [7].

\section{RESULTS AND DISCUSSION}

Fig. la compares the calculated correlation function for $\pi^{+}$pairs to the NA4h [8] data. The correlation functions vs. the magnitude of the four-momentum difference ( $q_{\text {inv }}=$ $\sqrt{-q \cdot q}$ are shown. In fig. I and in the figures which follow, the calculated correlation functions use only particles within a model of the NAMA acceptance $[2,8]$ in the low transverse momentum $\left(p_{T}\right)$ spectrometer sotting. For pions, this requires $p_{T}<0.4$ (ieV and $3.1<y<4.1$, where $y$ is the laboratory rapidity. For kanons, the acceptance is in the range $p t<0$.6(ieV and $y \approx 3$. The good agrement gives us some confidence is the technique and in the details of the model which influence the shape of the correlation finction.

When using an event gencrator to calculate correlation functions, we can examine not only the correlation functions, but the position distributions of the particles which give rise to the correlation function. The russ value of the transverse position ( $R T$ ) for pions $\left(4.9^{2} \pm .0^{2}\right.$ f fon) in the $\mathrm{N} \Lambda 41$ acceptance is significantly larger than the corresponding value

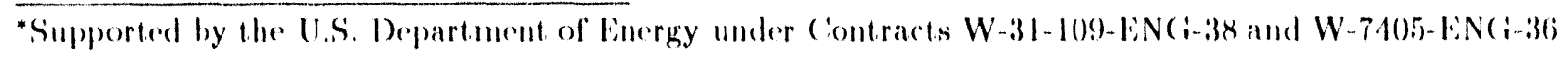



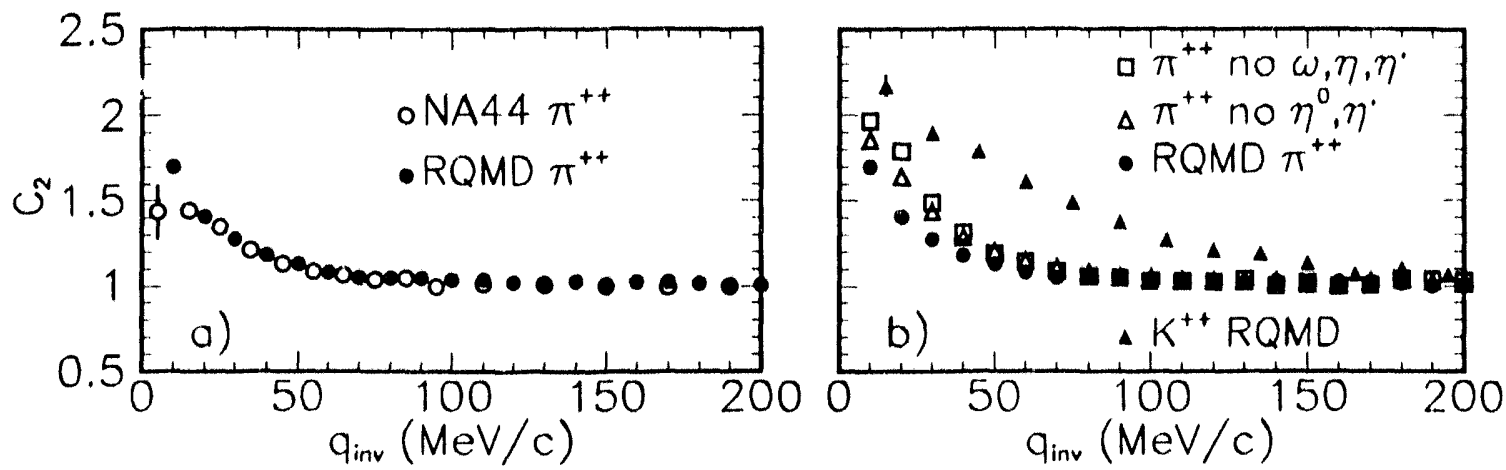

Figure 1. a) Comparison of pion correlation functions calculated from RQMD) with the NA44 data for 200 ( $\mathrm{c} \mathrm{V} / \mathrm{u} \mathrm{S}+\mathrm{Pb}$ collisions. b) Comparison of pion correlation functions calculated with all pions (from RQMD) and correlation functions omiting pions from 1 ) $\eta^{0}, \eta^{\prime}$ and 2) $\eta^{0}, \eta^{\prime}, \omega$ decay. $A$ calculated $k^{+}$correlation function is also shown.

for $\mathrm{K}^{+}(4.06 \pm .06 \mathrm{fm})$, where the uncertainties are statistical and the rms values use only particles with $R_{T}<20 \mathrm{fm}$; such a difference is also seen in the data [4].

Resonances influence the $R_{T}$ distributions and have important effects on correlation functions, especially for pions [9]. A resonance important for the shape of the pion correlation function is the $\omega(783)$, which produces about $14 \%$ of all charged pions with $2<y<4$ in R(QMI). With a long lifetime ( $I=8.43 \mathrm{McV}$ ) compared to the iifetime of the particle source, it contributes to a narrower and sharper peak in the correlation function. About 13\% of the pions in RQMD with $2<y<4$ come from $\eta^{0}$ and $\eta^{\prime}$ decay, whose widths (1.19 $\mathrm{keV}$ and $0.2 \mathrm{McV}$, respectively) produce components of the correlation function too narrow to measure. The result is a reduction in the intercept $(\lambda)$ of the measured correlation function. For $\pi^{+}$in the N $\Lambda 44$ acceptance (low $y^{2}$ ) the contribution from the $\omega(783)$ increases to $20 \%$ and the $\eta^{0}+\eta^{\prime}$ contribution increases to $21 \%$. For kaons, the most important long-lived resonance is the $K^{*}(892)$, however its width $(\mathrm{I}=50 \mathrm{MeV})$ is more closely matched to the widths of the kaon correlation functions. In addition, about $3 \%$ of $h^{+}$in RQMD with $2<y<4$ come from the $\phi(1020)(I=4.4$ $\mathrm{MeV}$ ) decay increasing to $4 \%$ in the NA44 acceptance. Fig. 2 shows the resonance contributions to the correlation functions as a function of $p T$. The fraction of pions from


Figure 2. From 200 ( $\mathrm{k} \times \mathrm{V} / 11 \mathrm{~S}+\mathrm{Ph}$ collisions in RQMI): The fraction of $\pi^{+}$in the region $2<y<4$ from $\omega(783)$ and the fraction from $\eta^{0}+\eta^{\prime}$ decay as a function of $p q$ (left). (O) the right, the fraction of $\mathrm{l}^{+}$from $\phi(1020)$ decay. 
resonance decays drops rapidly with increasing $p$. 'The resonance contribution is much less for $K^{+}$pointing out one advantage of kaon correlation functions [9].

Figure 16 shows the influence of these resonances on the calculated correlation functions. When the contributions from resonance decays are removed (open squares), the intercept approaches the ideal value of 2. Adding the pions from w(ts:3) decay (open triangles) reduees the intercept, and decreases the width of the correlation function - corresponding to a larger apparent source. Including pions from $n$ and $\|^{\prime}$ decay (solid circles) further reduces the intereept of the correlation function. 'This rase. with all resonance decays included, is compared to the data in figure la. For comparison, the calculated $\mathrm{h}^{+}$correlation function (solid triangles) is also shown. It has an intereept near 2 and is significantly wider (smaller source) than any of the pion correlation functions. This is expected since the $k+$ sourer is smaller than the pion source.

Fig. 3 compares correlation functions calculated from RQMI) for $K^{-}$and $k^{\text {t }}$ in the NA44 acceptance to the preliminary NAMA data [4, 5]. The agrerement is not as good as for pions. The calculated correlation functions are significantly wider than the data, therefore the apparent size of the kaon souree is larger in the dat a than in the calculations. The disagrerment is similar for both kaon charges. If the size differences (between $\pi$ and $k^{+}$) were cutirely doe to differences in rescattering cross sections, then $k^{+}$would reflect a smaller size than pions as is observed. for $h$ the expectations are not so straightforward. Like $\mathrm{K}^{+}$, their rescattering cross sections with other mesons are smaller than for pions. However, their rescattering cross sections on murleons are larger than the $\pi N$ cross sections. Differences in the measured $k^{+}$and $k^{-}$source sizes are expected if a significant fraction of the rescattering is from nucleons rather than mesons. In both the calculation and in the data the $R^{+}$and $K^{-}$correlation functions are similar to one another suggesting that collisions with mucleons do not dominate the dynamics.

Fig. 4 compares the measured and calculated $h^{+}$correlation function $[4,5]$ in more detail. In this case the correlation function is broken into three components [10, 11], defined relative to the direction of the average momentum of a pair of particles with momenta $\overrightarrow{p_{1}}$ and $\overrightarrow{p_{2}} \cdot\left(\vec{p}=\left(\overrightarrow{p_{1}}+\overrightarrow{p_{2}}\right) / 2\right)$ in a reference frame where the average longitudinal momentum of the pair is zero $(P z=0)$. In this frame $P$ is in the transwerse direction. The component of the momentum diflerence $\left(\vec{q}=\overrightarrow{p_{1}}-\overrightarrow{p_{2}}\right)$ along $\vec{p}$ is q timet the component along the beam axis is queam; the third component (qrate) is perpendicular to the first.


Figune:3. K (left) and $K^{+}$(right) correlation functions calculated from RQQMI) compared





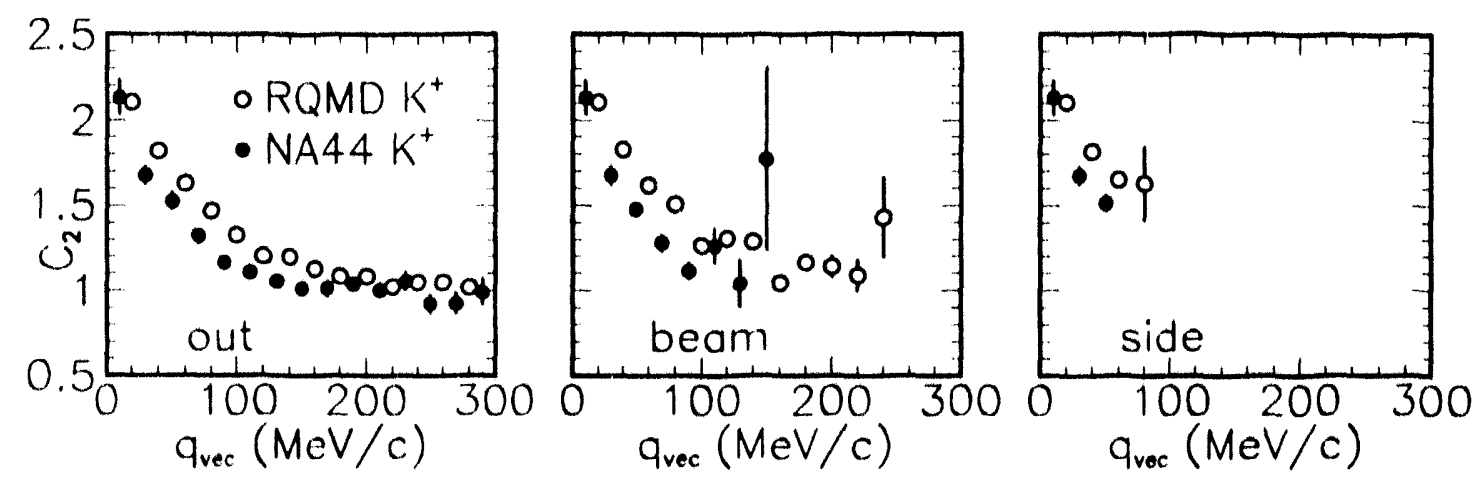

Figure 4. For 200 (ieV/uS $S+P b$, three components of $k^{+}$(right) correlation functions calculated from RQQMI) compared to preliminary NA44 datia.

two. In fig. 4, cach component of the correlation function is defined by plotting $\vec{q}$ while recpuiring the magnitude of the other two components to be less than $40 \mathrm{MeV} / \mathrm{c}$. The disagreement betwern the calculated and measured correlation functions is about the same in all directions.

\section{CONCIUUSIONS}

The predicted correlation functions for pion pairs are remarkably consistent with experiment. For katon pairs, the agrement with the data is not as good. However, both the calculations and the data show kaon source sizes which are smaller than those for pions and the $k^{+}$correlation functions are similar to the $k^{-}$correlation functions. 'The disagreement between the calculated and measured $k^{+}$correlation function is not confined to any directional component. Resonances have an important influence on the pion correlation functions. As shown in fig. 2, the influence of resonances on the pion correlation functions could be minimized by measuring at higher $p_{T}[12]$.

\section{REFERENCES}

1. P. Seyboth et al. (NA35 collaboration), Nucl. Phys. 1544 (1992) 2936.

2. M. Sarabura et al. (NAt4 collaboration), Nucl. Phys. A544 (1992) 125c.

3. J. P. Sullivan ol al., Mhys. Rev. laett. 70 (1993) 3000.

4. 'T. Humanic, this conference.

5. S. Pandery this ronference.

6. II. Sorge. II. Störker and W. (ireiner, Nucl Phys. A498 (1989) 5667; H. Sorge ot al., \%. Phys ( 47 (1999) (i22)

7. '. (sörgo, J. Kim myi, J. Bondorf, H. Heisellorg, and S. Pratt, Phys. lett. 13241

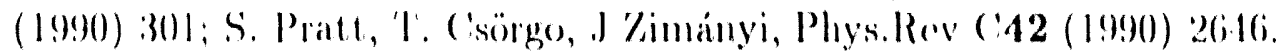

8. II. Boggild et al., Physs. Late. 13302 (1993) 510.

9. S. Padula, M. (iyulassy, Nucl. Phys. 13339, 378 (1990)).

10. S. Pratt, Phys. Rov. 1)33, 1:314 (1986).

11. (i. Bertsch, M. (iong, M. 'Tohyama, Phys. Rev. (37, Is!g (1988).

12. R. Jaymuti, this conference. 

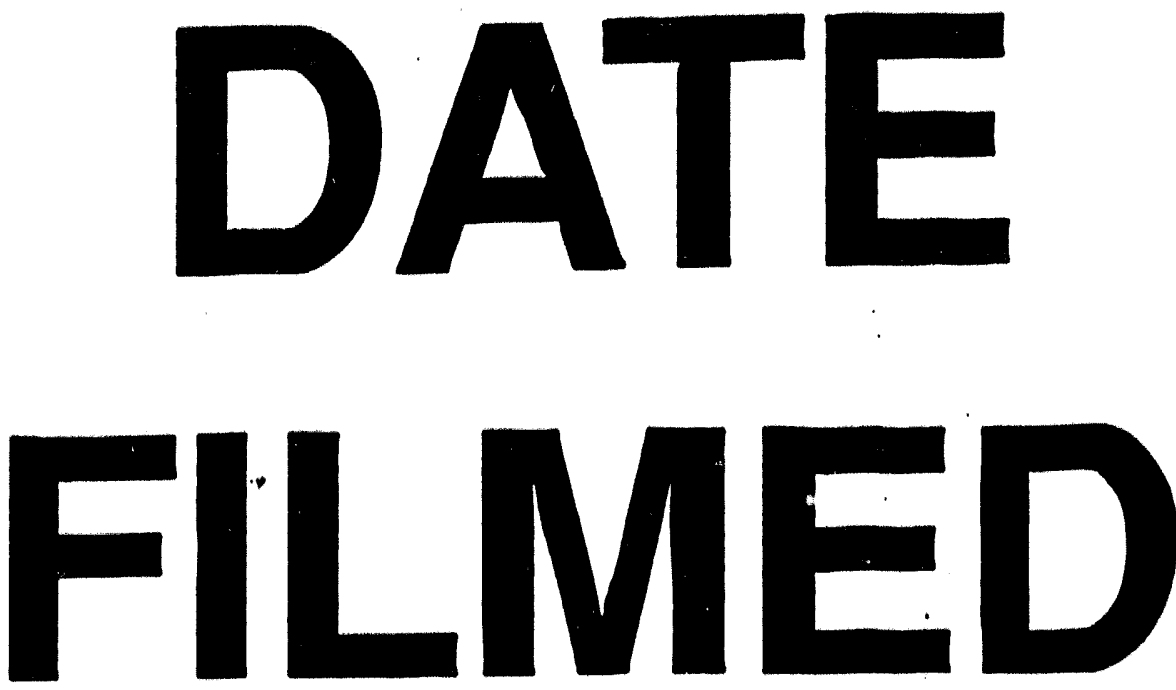

$12 / 13 / 93$
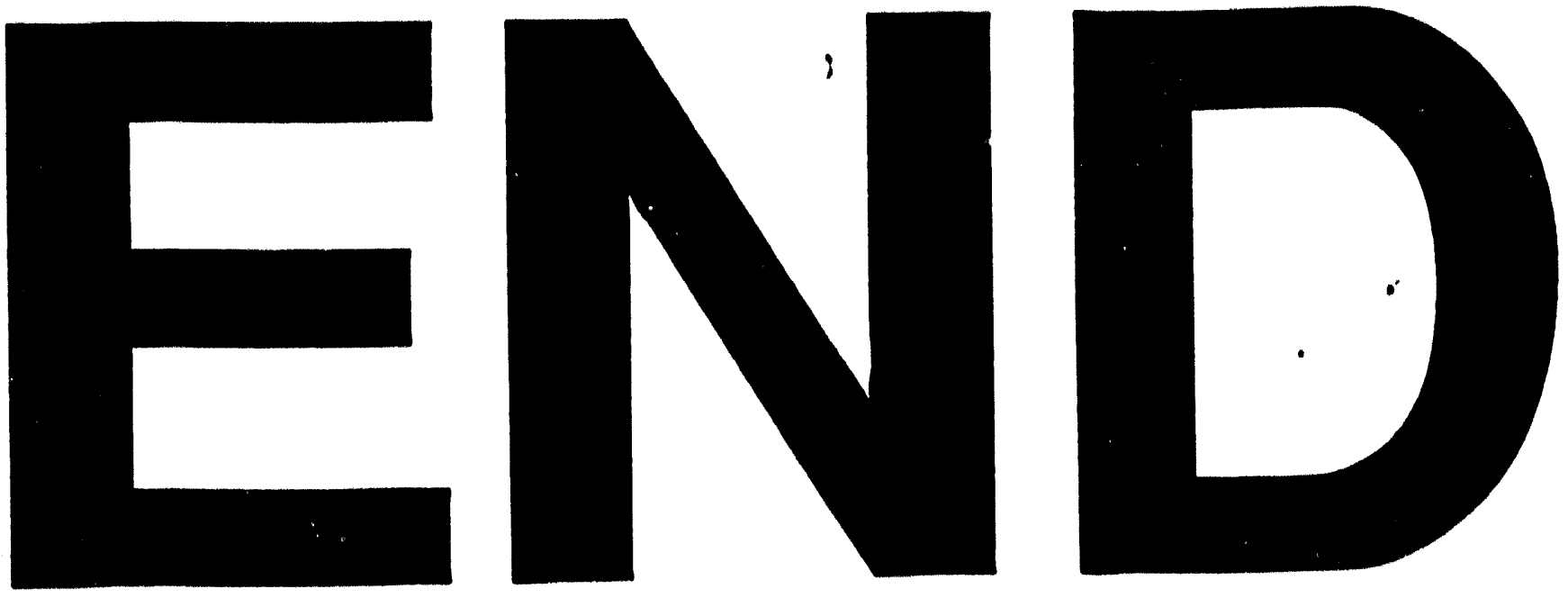

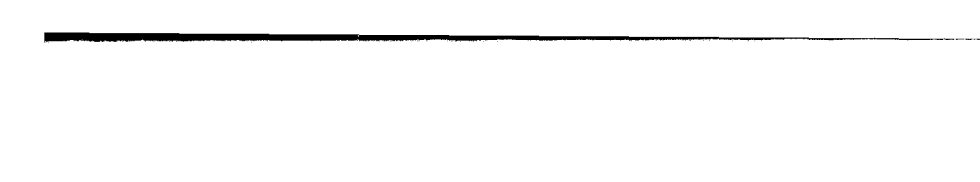